\title{
Sivers Asymmetry in $\pi N$ Drell-Yan process at COMPASS within TMD factorization
}

\section{Yaobin Fu}

School of Physics and Engineering, Zhengzhou University, Zhengzhou, Henan 450001, China

E-mail: fu_vaobind163.com

\section{Shi-Chen Xue}

School of Physics and Engineering, Zhengzhou University, Zhengzhou, Henan 450001, China

E-mail: Shichen.gs.zzu.edu.cn

\section{Xiaoyu Wang* ${ }^{\dagger}$}

School of Physics and Engineering, Zhengzhou University, Zhengzhou, Henan 450001, China

E-mail: 区iaoyuwangezzu.edu.cn

\section{Zhun Lu}

School of Physics, Southeast University, Nanjing 211189, China

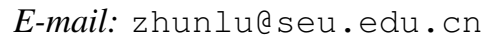

\section{Ivan Schmidt}

Departamento de Física, Universidad Técnica Federico Santa María, and Centro

Científico-Tecnológico de Valparaíso, Casilla 110-V, Valparaíso, Chile

We study the differential cross section in the unpolarized $\pi^{-} N$ Drell-Yan process, using transverse momentum dependent factorization up to next-to-logarithmic order of QCD and extract the nonperturbative Sudakov form factor for the pion in the evolution formalism of the unpolarized TMD distribution function, by fitting the experimental data collected by the E615 Collaboration at Fermilab. With the extracted Sudakov factor, we investigate the Sivers asymmetry in the pion-induced single polarized Drell-Yan process in the theoretical framework of the transverse momentum dependent factorization up to next-to-leading logarithmic order of QCD. Within the TMD evolution formalism of parton distribution functions, the extracted nonperturbative Sudakov form factor for the pion distribution functions as well as the one for the Sivers function of the proton are applied to numerically estimate the Sivers asymmetry in the $\pi^{-} p$ Drell-Yan at the kinematics of the COMPASS at CERN.

XXVII International Workshop on Deep-Inelastic Scattering and Related Subjects - DIS2019

8-12 April, 2019

Torino, Italy

\footnotetext{
*Speaker.

${ }^{\dagger}$ This work is partially supported by the NSFC (China) grant 11575043 and grant 11847217 , by the Fundamental Research Funds for the Central Universities of China, by Fondecyt (Chile) grants 1140390 and FB-0821. X. Wang is supported by the Scientific Research Foundation of Graduate School of Southeast University (Grants No. YBJJ1667) and by the China Postdoctoral Science Foundation under Grant No. 2018M640680.
} 


\section{Introduction}

One of the main goals of the COMPASS Drell-Yan program is to measure the Sivers asymmetry or the Sivers function with high precision. Sivers function [U] is a transverse momentum dependent (TMD) parton distribution function (PDF) describing the asymmetric density of unpolarized quarks inside a transversely polarized nucleon. It can give rise to the transversely single-spin asymmetry in various high energy scattering processes. Because of its T-odd property, QCD predicts that the sign of the Sivers function changes in SIDIS with respect to the Drell-Yan process [, [1, 团]. The verification of this sign change is one of the most fundamental tests of our understanding of the QCD dynamics and the factorization scheme.

The main purpose of this work is to apply the TMD factorization to present a detailed phenomenological analysis of the Sivers asymmetry in the pion induced Drell-Yan process that is accessible at COMPASS [5]. Particularly, we take into account the TMD evolution for both the pion distribution functions and the proton distribution functions. We perform the extraction of the nonperturbative Sudakov form factor for the unpolarized pion distribution funtion $f_{1, q / \pi}\left(x_{\pi}, k_{T}^{2}\right)$ to evolve $f_{1, q / \pi}\left(x_{\pi}, k_{T}^{2}\right)$. As for the TMD evolution effect for the distributions of the proton, we apply the existed parametrization of the nonperturbative Sudakov form factor for the unpolarized distribution function and Sivers function.

\section{Extracting the nonperturbative Sudakov form factor for the unpolarized pion TMD distribution}

In the unpolarized $\pi^{-} P$ Drell-Yan process $\pi\left(P_{\pi}\right)+p\left(P_{p}\right) \longrightarrow \gamma^{*}(q)+X \longrightarrow l^{+}(\ell)+l^{-}\left(\ell^{\prime}\right)+$ $X$, the differential cross section can be expressed as a $b$-dependent function ( $\boldsymbol{b}$ is conjugate to $\boldsymbol{q}_{\perp}$ via Fourier Transformation) formulated in TMD factorization since the simplicity by introducing the coordinate $\boldsymbol{b}$-space and then transform back to transverse momentum space to represent the experimental observables [焑] $\frac{d^{4} \sigma}{d Q^{2} d y d^{2} \boldsymbol{q}_{\perp}}=\sigma_{0} \int \frac{d^{2} b}{(2 \pi)^{2}} e^{i \overrightarrow{\boldsymbol{q}}_{\perp}} \cdot \overrightarrow{\boldsymbol{b}}_{W_{U U}}(Q ; b)+Y_{U U}\left(Q, q_{\perp}\right) \cdot \sigma_{0}=\frac{4 \pi \alpha_{e m}^{2}}{3 N_{C} S Q^{2}}$ is the cross section at tree level, structure function $\widetilde{W}(Q ; b)$ contains all-order resummation results and dominates at low $q_{\perp} \ll Q$ value, while the term $Y_{U U}$ provides the necessary correction at moderate $q_{\perp} \sim Q$ values (we use the tilde to denote $b$-space terms). We will focus on the region $q_{\perp} \ll Q$ and neglect the $Y$-term.

$\widetilde{W}(Q ; b)$ can be expressed as [0]

$$
\widetilde{W}_{U U}(Q ; b)=H_{U U}(Q ; \mu) \sum_{q, \bar{q}} e_{q}^{2} \tilde{f}_{1 \bar{q} / \pi}^{\mathrm{sub}}\left(x_{\pi}, b ; \mu, \zeta_{F}\right) \tilde{f}_{1 q / p}^{\mathrm{sub}}\left(x_{p}, b ; \mu, \zeta_{F}\right)
$$

where $\tilde{f}_{q / H}^{\text {sub }}$ is the subtracted distribution function in $b$-space with the soft factor subtracted, $H_{U U}(Q ; \mu)$ is the hard scattering factor, $\mu$ is renormalization scale in the case of collinear PDFs, and $\zeta_{F}$ denotes an energy scale related to the cutoff of the TMD distributions. The TMD evolution for the $\zeta_{F}$ and $\mu$ dependence of TMD PDFs is encoded in Collins-Soper (CS) [ [ $]$ ] and the renormalization group equation. The overall solution structure for $\tilde{f}_{1}\left(x, b ; \mu, \zeta_{F}\right)$ is the same as that for the Sudakov form factor as $\exp (-S)^{1}$

$$
\tilde{f}(x, b, Q)=\mathscr{F} \times e^{-S} \times \tilde{f}(x, b, \mu) .
$$

\footnotetext{
${ }^{1}$ Hereafter, we set $\mu=\sqrt{\zeta_{F}}=Q$ and express $f\left(x, b ; \mu=Q, \zeta_{F}=Q^{2}\right)$ as $f(x, b ; Q)$ for simplicity.
} 
Here, $\mathscr{F}$ is the hard factor dependent on the scheme that we choose. A parameter $b_{\max }$ with typical value chosen around $1 \mathrm{GeV}^{-1}$ is introduced to serve as boundary between the perturbative and nonperturbative regions. $b$-dependent function $b_{*}=b / \sqrt{1+b^{2} / b_{\max }^{2}}$ [ [ ] guarantees $b_{*}$ is always at the perturbative region. In the small $b$ region $1 / Q \ll b \ll 1 / \Lambda$, the TMDs at fixed energy $\mu$ can be expressed as a convolution of perturbatively calculable hard coefficients and the corresponding collinear PDFs [Q, ए0] $f_{1 q / H}(x, b ; \mu)=\sum_{i} C_{q \leftarrow i} \otimes f_{1}^{i / H}(x, \mu) . f_{1}^{i / H}(\xi, \mu)$ is the corresponding collinear PDF at the energy scale $\mu$, which can be a dynamic scale related to $b_{*}$ by $\mu_{b}=c_{0} / b_{*}$, with $c_{0}=2 e^{-\gamma_{E}}$ and $\gamma_{E} \approx 0.577$, the Euler Constant [Q] ]. The Sudakov form factor $S$ in Eq. (2.2) can be separated into a perturbatively calculable part and a nonperturbative part $S=S_{\text {pert }}+S_{\mathrm{NP}}$. The perturbative part $S_{\text {pert }}$ can be expanded $\alpha_{s} / \pi$ series, which we take up to the accuracy of next-to-leading-logarithmic (NLL) order. For the nonperturbative form factor $S_{\mathrm{NP}}$, a general parametrization associated with the TMD PDF for one of the initial protons has the form [ए]] $S_{\mathrm{NP}}^{f_{1}^{q / p}}(Q, b)=\frac{g_{1}}{2} b^{2}+\frac{g_{2}}{2} \ln \frac{b}{b_{*}} \ln \frac{Q}{Q_{0}}$. The parameters are fitted from the nucleon-nucleon Drell-Yan process data at the initial scale $Q_{0}^{2}=2.4 \mathrm{GeV}^{2}$ and $b_{\max }=1.5 \mathrm{GeV}^{-1}$, with the results being $g_{1}=0.212, g_{2}=0.84$ [W]. The nonperturbative Sudakov form factor for the pion TMD distributions has never been obtained. Here we assume that it has the same structure as for the proton TMD distributions, with parameters $g_{1}^{\pi}$ and $g_{2}^{\pi}$

$$
S_{\mathrm{NP}}^{f_{1}^{q / \pi}}=g_{1}^{\pi} b^{2}+g_{2}^{\pi} \ln \frac{b}{b_{*}} \ln \frac{Q}{Q_{0}} .
$$

After dealing with the scheme dependence of the hard factors $H, \mathscr{F}$ and $C$, we can reach the structure function in $b$-space as

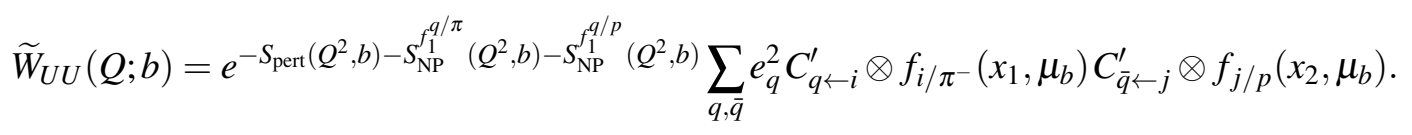

Performing the Fourier Transformation, we can get the differential cross section

$$
\frac{d^{4} \sigma}{d Q^{2} d y d^{2} \boldsymbol{q}_{\perp}}=\sigma_{0} \int_{0}^{\infty} \frac{d b b}{2 \pi} J_{0}\left(q_{\perp} b\right) \times \widetilde{W}_{U U}(Q ; b) .
$$

Fitting the differential cross section with the E615 experimental data [12], ㅈ] by minimizing chisquare, we obtain the following values for the two parameters:

$$
g_{1}^{\pi}=0.082 \pm 0.022, \quad g_{2}^{\pi}=0.394 \pm 0.103, \text { with } \chi^{2} / \text { d.o.f }=1.64 .
$$

Applying the extracted $S_{\mathrm{NP}}^{f_{1}^{q / \pi}}$, we calculate the transverse momentum spectrum of dimuon pairs produced in unpolarized pion-nucleon Drell-Yan processes at the COMPASS kinematics and compare with the COMPASS data [5] to verify our extraction, with the results plotted in Fig. [. Comparing the two curves, we find that our theoretical estimate on the $q_{\perp}$ distribution of the dimuon agrees with the COMPASS data fairly well in the small $q_{\perp}$ region, in both the shape and the size. This validates our extraction of the nonperturbative Sudakov form factor for the pion distribution $f_{1 \pi}$, within the TMD factorization and indicates the framework applied can also be extended to the study of the azimuthal asymmetries in the $\pi N$ Drell-Yan process. 


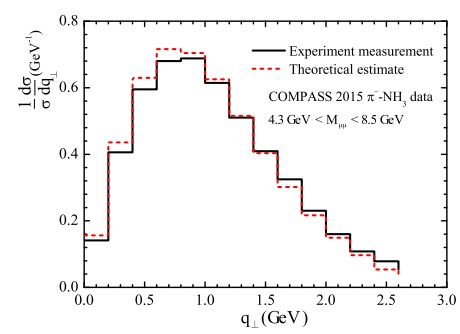

Figure 1: The transverse spectrum of lepton pair production in the unpolarized pion-nucleon Drell-Yan process, with an $\mathrm{NH}_{3}$ target at COMPASS. The dashed line is our theoretical calculation using the extracted Sudakov form factor for the pion TMD PDF. The solid line shows the experimental measurement at COMPASS [回].

\section{Sivers Asymmetry in the pion induced Drell-Yan process at COMPASS}

The transverse single spin asymmetry for the unpolarized $\pi^{-}$scattering off the transversely polarized proton Drell-Yan process can be defined as $A_{U T}=\frac{d^{4} \Delta \sigma}{d Q^{2} d y d^{2} \boldsymbol{q}_{\perp}} / \frac{d^{4} \sigma}{d Q^{2} d y d^{2} \boldsymbol{q}_{\perp}}$ [ए1]] with $\frac{d^{4} \sigma}{d Q^{2} d y d^{2} \boldsymbol{q}_{\perp}}$ for the spin-averaged differential cross section written in Eq. (2.5), while $\frac{d^{4} \Delta \sigma}{d Q^{2} d y d^{2} \boldsymbol{q}_{\perp}}$ for the spin-dependent differential cross section, which can be expressed similar to the unpolarized case as $\frac{d^{4} \Delta \sigma}{d Q^{2} d y d^{2} \boldsymbol{q}_{\perp}}=\sigma_{0} \varepsilon_{\perp}^{\alpha \beta} S_{\perp}^{\alpha} \int \frac{d^{2} b}{(2 \pi)^{2}} e^{i \vec{q}_{\perp}} \cdot \overrightarrow{\boldsymbol{b}}_{W T} \widetilde{W}_{U T}^{\beta}(Q ; b)+Y_{U T}^{\beta}\left(Q, q_{\perp}\right)$ [155, [4], [6]. Similarly, we will only consider the $\widetilde{W}_{U T}^{\beta}$-terms, which can be written according to the TMD factorization as [6, []]

$$
\widetilde{W}_{U T}^{\alpha}(Q ; b)=H_{U T}(Q ; \mu) \sum_{q, \bar{q}} e_{q}^{2} \tilde{f}_{1, \bar{q} / \pi}\left(x_{\pi}, b ; \mu, \zeta_{F}\right) \tilde{f}_{1 T, q / p}^{\perp \alpha(\mathrm{DY})}\left(x_{p}, b ; \mu, \zeta_{F}\right)
$$

$\tilde{f}_{1, q / \pi}$ and $\tilde{f}_{1 T, q / p}^{\perp \alpha}$ stand for the unpolarized distribution function of pion meson and the Siver$\mathrm{s}$ function for proton in the $b$-space with the soft factor subtracted in the definition of the TMD distribution functions, respectively. The latter one has the definition as $\tilde{f}_{1 T q / p}^{\perp \alpha(\mathrm{DY})}(x, b ; \mu)=$ $\int d^{2} \boldsymbol{k}_{\perp} e^{-i \vec{k}_{\perp} \cdot \overrightarrow{\boldsymbol{b}}} \frac{k_{\perp}^{\alpha}}{\bar{M}_{p}} f_{1 T, q / p}^{\perp(\mathrm{DY})}\left(x, \boldsymbol{k}_{\perp} ; \mu\right)$ [ए4]]. The superscript DY denotes that the quark Sivers function is the one in the Drell-Yan process, and it satisfies the relation $f_{1 T, q / p}^{\perp(\mathrm{DY})}=-f_{1 T, q / p}^{\perp \text { (DIS) }}$. The solution structure of the evolution equations for the Sivers function is exactly the same as that in Eq. (2.2) with the same perturbative Sudakov form factor. The nonperturbative Sudakov form factor $S_{\mathrm{NP}}$ has been proposed in Ref. [14] for the Sivers function as $S_{\mathrm{NP}}^{\mathrm{Siv}}=\left(g_{1}^{\mathrm{Siv}}+g_{2}^{\mathrm{Siv}} \ln \frac{Q}{Q_{0}}\right) b^{2}$ with $g_{1}^{\text {Siv }}$ relevant to the averaged intrinsic transverse momenta squared $g_{1}^{\mathrm{Siv}}=\left\langle k_{s \perp}^{2}\right\rangle_{Q_{0}} / 4=0.071 \mathrm{GeV}^{2}$ and $g_{2}^{\mathrm{Siv}}$ being $\frac{1}{2} g_{2}=0.08 \mathrm{GeV}^{2}$. Similar to the unpolarized distribution function in the low $b$ region, the Sivers function $\tilde{f}_{1 T q / p}^{\perp \alpha(\mathrm{DY})}$ can be also expressed as the convolution of perturbatively calculable hard coefficients and the corresponding collinear correlation functions as [ए6, ए5]

$$
\tilde{f}_{1 T q / p}^{\perp \alpha(\mathrm{DY})}(x, b ; \mu)=\left(\frac{-i b^{\alpha}}{2}\right) \sum_{i} \Delta C_{q \leftarrow i}^{T} \otimes f_{i / p}^{(3)}\left(x^{\prime}, x^{\prime \prime} ; \mu\right) .
$$

$\Delta C_{q \leftarrow i}^{T}$ stands for the hard coefficients and $f_{i / p}^{(3)}\left(x^{\prime}, x^{\prime \prime}\right)$ denotes the twist-three quark-gluon-quark or trigluon correlation functions, among which the Qiu-Sterman function $T_{q, F}(x, x)$ [ए], ㅍ, एव] may provide the main contribution to the single spin asymmetry. It is proportional to the first transverse 
moment of the Sivers function $f_{1 T q / p}^{\perp(1)}(x)$ [ए6, ए15] $T_{q, F}(x, x)=2 M_{p} f_{1 T q / p}^{\perp(1) \mathrm{DY}}(x)$. Consistent with the choice of the COMPASS experiments [[]] and solving the scheme-dependent problem on the hard scattering factor, the spin-dependent differential cross section can be rewritten as

$\frac{d^{4} \Delta \sigma}{d Q^{2} d y d^{2} \boldsymbol{q}_{\perp}}=\frac{\sigma_{0}}{4 \pi} \int_{0}^{\infty} d b b^{2} J_{1}\left(q_{\perp} b\right) \sum_{q, i, j} e_{q}^{2} \Delta C_{q \leftarrow i}^{T} \otimes T_{i, F}\left(x_{p}, x_{p} ; \mu_{b}\right) C_{\bar{q} \leftarrow j} \otimes f_{1, j / \pi}\left(x_{\pi}, \mu_{b}\right) e^{-\left(S_{\mathrm{NP}}^{\mathrm{Siv}}+S_{\mathrm{NP}}^{f_{1 q} / \pi}+S_{\mathrm{P}}\right)}$.

We adopt the NLO set of the CT10 parametrization [LD] (central PDF set) and the NLO SMRS parametrization [2] for the unpolarized distribution function $f_{1}(x)$ of the proton and pion meson, respectively. For the Qiu-Sterman functions $T_{q, F}(x, x ; \mu)$, we adopt the parameterization [14] extracted from the Sivers asymmetry in SIDIS. There are two different approaches adopted to deal with the scale dependence of $T_{q, F}$ for comparison: set 1 assumes that $T_{q, F}$ is proportional to the usual unpolarized collinear PDF at any scale, namely, $T_{q, F}$ follows the DGLAP evolution as that of $f_{1}$, like the choice in Ref. [14], while set 2 adopts the parameterizations at the initial scale $\left(Q_{0}^{2}=2.4 \mathrm{GeV}^{2}\right)$ then evolves it to another scale $Q$ through QCD evolution via an approximate evolution kernel (only including the homogenous terms) $P_{q q}^{\mathrm{QS}} \approx P_{q q}^{f_{1}}-\frac{N_{c}}{2} \frac{1+z^{2}}{1-z}-N_{c} \delta(1-z)$.
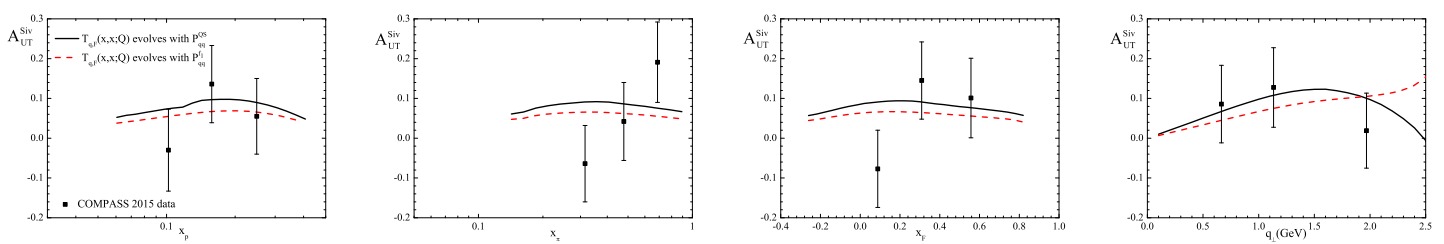

Figure 2: The Sivers asymmetry for $\pi^{-}$scattering off transversely polarized proton Drell-Yan process. The dashed lines represent Sivers asymmetry with the Qiu-Sterman functions $T_{q, F}(x, x ; Q)$ being proportional to the unpolarized PDF $f_{1}(x, Q)$ (Set 1$)$. The solid lines depict Sivers asymmetry considering Qiu-Sterman functions evolving through the splitting function (Set 2).

The numerical estimate of the Sivers asymmetry $A_{U T}^{\text {Siv }}$ in the $\pi^{-} p$ Drell-Yan at the kinematics of COMPASS is plotted in Fig. \. As a comparison, the experimental data measured by the COMPASS Collaboration [5] with error bars is also shown in Fig. [1. One can find that in all the cases the Sivers asymmetry in the $\pi^{-} p$ Drell-Yan from our calculation is positive with the size around 0.05 to 0.10. It is consistent with the COMPASS measurement shown in Fig.5 of Ref. [5] within the uncertainties of the asymmetry. We also find that the asymmetry from the Sivers function in set 2 is more sizable than the one from set 1 , and is more closer to the central values of the asymmetry measured by COMPASS. This is because that the Sivers function in set 2 is larger than the Sivers function in set 1 . Furthermore, compared to the asymmetry from set 1, the asymmetry from set 2 has a fall at larger $q_{\perp}$, which is more compatible to the shape of $q_{\perp}$-dependent asymmetry of measured by the COMPASS Collaboration.

\section{Conclusion}

In this work, we applied the formalism of the TMD factorization to study the Sivers asymmetry in the pion induced Drell-Yan process that is accessible at COMPASS. We took into account the 
TMD evolution of the pion distribution functions as well as the proton distributions. For the former one, we parameterized the Sudakov form factor in a form analogous to that of the proton TMD distribution and performed a fit using the experimental data at E615. Adopting the extracted results, we calculated the transverse momentum spectrum of dimuon pairs produced in unpolarized pionnucleon Drell-Yan processes at the COMPASS kinematics, and compared it with the COMPASS data to verify our extraction. As for the TMD evolution of the proton distributions, we adopted the existed parametrization of the nonperturbative Sudakov form factor. We applied two different ways to treat the energy dependence of the Qiu-Sterman function which is proportional to first $k_{\perp}$ moment of the Sivers function. We then calculated the Sivers asymmetry in $\pi p$ Drell-Yan process at COMPASS. We find that the Sivers asymmetry calculated from the TMD evolution formalism is consistent with the COMPASS measurement. Furthermore, different treatments on the scale dependence of the Qiu-Sterman function yield different sizes and shapes of the asymmetries, which shows that the scale dependence of the Qiu-Sterman function will play a role in the interpretation of the experimental data, and it should also be considered in the phenomenological studies.

\section{References}

[1] D. W. Sivers, Phys. Rev. D 41, 83 (1990).

[2] S. J. Brodsky, D. S. Hwang and I. Schmidt, Phys. Lett. B 530, 99 (2002).

[3] S. J. Brodsky, D. S. Hwang and I. Schmidt, Nucl. Phys. B642, 344 (2002).

[4] J. C. Collins, Phys. Lett. B 536, 43 (2002).

[5] M. Aghasyan et al. (COMPASS Collaboration), Phys. Rev. Lett. 119, 112002 (2017).

[6] J. C. Collins, D. E. Soper and G. F. Sterman, Nucl. Phys. B250, 199 (1985).

[7] A. Prokudin, P. Sun and F. Yuan, Phys. Lett. B 750, 533 (2015).

[8] J. Collins, Foundations of perturbative QCD, Cambridge University Press, 2013.

[9] J. C. Collins and D. E. Soper, Nucl. Phys. B193, 381 (1981), Erratum: [Nucl. Phys. B213, 545 (1983)].

[10] A. Bacchetta and A. Prokudin, Nucl. Phys. B875, 536 (2013).

[11] P. Sun, J. Isaacson, C.-P. Yuan and F. Yuan, arXiv:1406.3073 [hep-ph].

[12] J. S. Conway et al., Phys. Rev. D 39, 92 (1989).

[13] W. J. Stirling and M. R. Whalley, J. Phys. G19 D1 (1993).

[14] M. G. Echevarria, A. Idilbi, Z. B. Kang and I. Vitev, Phys. Rev. D 89, 074013 (2014).

[15] Z. B. Kang, B. W. Xiao and F. Yuan, Phys. Rev. Lett. 107, 152002 (2011).

[16] P. Sun and F. Yuan, Phys. Rev. D 88, 114012 (2013).

[17] J. w. Qiu and G. F. Sterman, Phys. Rev. Lett. 67, 2264 (1991).

[18] J. w. Qiu and G. F. Sterman, Nucl. Phys. B378, 52 (1992).

[19] J. w. Qiu and G. F. Sterman, Phys. Rev. D 59, 014004 (1999).

[20] H. L. Lai, M. Guzzi, J. Huston, Z. Li, P. M. Nadolsky, J. Pumplin and C.-P. Yuan, Phys. Rev. D 82, 074024 (2010).

[21] P. J. Sutton, A. D. Martin, R. G. Roberts and W. J. Stirling, Phys. Rev. D 45, 2349 (1992). 